\title{
Téoros
}

Revue de recherche en tourisme

\section{Les modèles d'impact des événements touristiques}

\section{Faouzi Rassi}

Volume 7, numéro 3, novembre 1988

Économie du tourisme

URI : https://id.erudit.org/iderudit/1080383ar

DOI : https://doi.org/10.7202/1080383ar

Aller au sommaire du numéro

Éditeur(s)

Université du Québec à Montréal

ISSN

0712-8657 (imprimé)

1923-2705 (numérique)

Découvrir la revue

Citer cet article

Rassi, F. (1988). Les modèles d'impact des événements touristiques. Téoros, 7(3), 36-39. https://doi.org/10.7202/1080383ar d'utilisation que vous pouvez consulter en ligne.

https://apropos.erudit.org/fr/usagers/politique-dutilisation/ 


\section{Les modèles d'impact des événements touristiques}

\section{Introduction}

L'évaluation des effets des grands événements touristiques s'effectue, en général, à l'aide de modèles quantitatifs. Elle a pour objet d'établir les différentes catégories de coûts et d'avantages, de les chiffrer et d'en déterminer les résultats monétaires. Le calcul et l'analyse des effets et des dépenses additionnelles découlant de l'implantation d'un nouveau projet touristique d'envergure, se limitent habituellement à la région avoisinante. Les résultats recherchés sont les revenus supplémentaires engendrés par le nouveau projet, l'investissement nouveau, l'emploi créé et les infrastructures développées.

La plupart des études d'impact de grands événements touristiques se basent sur une enquête sur place c'est-à-dire dans la région immédiate où se réalisent ces événements. L'enquête est menée auprès des touristes afin d'evaluer, entre autres, les dépenses par catégories et par origine des touristes, la durée du séjour, le mode d'hébergement et la préférence en matière d'activités récréatives.

Toutefois, l'impact des grands événements touristiques ne se limite pas au seul plan économique. Il s'étend au plan socio-culturel, au plan politique et, sur le plan géographique, à la province ou même à l'ensemble du pays.

Par ailleurs, les méthodes d'analyse coûtsbénéfices ainsi que les modèles d'échanges inter-industriels peuvent étre complétés par les méthodes appliquées aux sciences de la gestion. Ces dernières regroupent et traitent des données à la fois quantitatives et qualitatives.

\section{Définitions}

Tout modèle est une représentation simplifiée d'une réalité plus complexe; il peut être de nature qualitative ou quantitative; d'equilibre partiel ou d'équilibre général; de nature macro-économique ou micro-économique; de nature statique ou dynamique, de nature purement théorique ou élaboré à partir d'études empiriques. Les sciences de la gestion utilisent souvent des modeles, depuis seulement un quart de siècle.

Les grands événements touristiques sont soit de nature récurrente à durée limitée soit réalisés une fois pour toute avec pour conséquence un impact majeur selon Brent Ritchie (1). L'auteur ajoute que ces événements améliorent a court et a long terme la prise de conscience, l'attention et la rentabilité d"une destination touristique. Ces événements ont des caractéristiques propres qui leurs sont uniques et qui garantissent leur succès. Les attractions et événements touristiques marquants suivants illustrent la nature et l'importance de telles réalisations:

- les expositions internationales: l'Exposition de Montréal en 1967 (Expo 67);

- les événements sportifs d'envergure: les Jeux Olympiques d'hiver de Calgary en 1988: la Coupe du Monde de football en Espagne en 1982;

- l'aménagement et la création de parcs nationaux (les parcs nationaux au Canada; les parcs nationaux au Kenya).

\section{Les modèles de nature qualitative}

Les modèles qualitatifs tentent de cerner et d'expliquer le comportement du consommateur. Ce genre d'analyse permet d'établir des prévisions en matière d'achat des ménages, de mieux comprendre le processus de décision du consommateur. Les politiques gouvernementales, la publicité des entreprises du secteur privé sont en partie axées sur les résultats obtenus par les modèles qualitatifs. Les études d'impact d'évênements d'envergure sont d'autant plus utiles à la décision des différents intervenants (différents paliers de gouvernement, le secteur privé, les autorités locales ou régionales) que la qualité de l'analyse qualitative est grande. James M. Rovelstad ${ }^{(2)}$ présente un exemple de modèle qualitatif appliqué au tourisme et pouvant servir à des fins de planification et de marketing. L'auteur se propose de développer une meilleure intuition, une plus grande familiarité des raisons qui morivent les touristes dans le choix d whe destination donnee, afin de mieux cerner l'impact de leur décision. Le modèle fut appliqué à l'État de la Virginie de 1'Ouest en regroupant les réponses obtenues aux questions posées à l'occasion d'une enquête auprès des touristes. Il s'agissait de déterminer les facteurs les plus importants dans le choix d'une destination de vacanciers. Quatre groupes de facteurs sont retenus:

- le facteur "qualité esthétique" (E):

- le rapport temps/coúts (C);

- la qualité de vie (Q):

- les accommodations (A). 
Le modèle, quoique de nature qualitative, peut être présenté par une relation mathématique:

$T=f(E, C, Q, A)$

où:

$T=$ nombre de touristes pour une destination donnée

$\mathrm{E}=$ paysages, sites historiques, détente

$\mathrm{C}=$ temps et coûts requis pour atteindre une destination

$Q=$ manque de pollution, l'accueil des résidents de la destination touristique

$\mathrm{A}=$ logements, activités récréatives

Les résultats de l'enquête indiquent l'importance relative de ces quatre groupes de facteurs:

$$
\mathrm{A}>\mathrm{E}>\mathrm{C}>\mathrm{Q}
$$

\section{Les modèles de nature quantitative}

\section{Les bénéfices}

Un projet touristique de grande envergure comme la création d'un parc national ou aussi la réalisation de Jeux Olympiques au Canada entraine pour la région ou la province concernée des benéfices et des coûts qu'il convient de préciser. Les catégories de bénéfices que peut retirer une région ou une province sont les suivantes:

a) Les bénéficies de la préservation: la protection du milieu naturel pour assurer la prétservation en tant que telle (sites, flore, faune, ...); la préservation de l'environnement pour assurer la détente et la récréation; la préservation pour des motifs économiques tel que le développement de l'activité touristique et la création d'emploi.

\section{b) Les benefices de la recreation}

c) Les benefices des iransferts interrégionaux: ce sont des fonds transférés par le gouvemement féderal canadien au gouvernement provincial où se situe le parc national de grande envergure. L'analyse economique ne retient que les transferts monétaires qui suscitent un surcroît d'activité dans une province et qui sont générateurs de nouveaux emplois et de revenus supplémentaires.

Notons cependant que les transferts interrégionaux nets sont retenus dans ce genre d'analyse et mesurent la contribution nette de l'État fédéral aux projets touristiques d'envergure réalisés dans une région, dans une province donnée: contributions en dépenses de capital ou en dépenses d'exploitation courante. En effet, les transferts interrégionaux bruts doivent être calculés abstraction faite de la contribution fiscale des contribuables de la région ou de la province aux revenus fédéraux. Le montant net des transferts interfégionaux ainsi calculé doit tenir compte des fuites que représentent les achats de biens d'équipement et de consommation importés par la région ou la province pour satisfaire les besoins de construction et de fonctionnement du pare national. Le montant nét ainsi obtenu, c'est-à-dire après déduction des impóts et des fuites, doit être mulitiplié par le multiplicateur des dépenses régionales ou provinciales afin d'établir les effets expansionnistes des dépenses fédérales sur l'ensemble de l'activité économique, sur les revenus et l'emploi ainsi que sur la fiscalité provinciale par exemple.

\section{d) Les benefices découlant des flux de depen- ses touristiques}

\section{d. 1- Le traitement des fux touristiques par un modèle inter-industriel national ou pro- vincial}

Les flux touristiques engendrés par un grand événement sont d'autant plus difficiles à cerner que les statistiques touristiques officielles concernant la région ou de la province étudiée ne sont pas disponibles.

Les résultats d'une enquête sur place auprès d'individus ou d'unités familiales constituent la base des prévisions de la consommation touristique par type d'hébergement, par structure de dépenses et par zone d'origine de touristes. Il s'agit d'elaborer ensuite un modèle de prévision des flux touristiques annuels par type d'hébergement pour un horizon donné de dix ans par exemple, afin $\mathrm{d}^{*} e n$ déduire les conséquences sur les investissements nécessaires à la satisfaction de la demande par catégorie d'hébergement. Notons que l'analyse par type de fluxhébergement est supérieure à celle par activité récréative, car elle permet d'évaluer l'impact des prévisions de la demande touristique sur les investissements concernant l'hébergement. Cette catégorie d'investissements est en général la plus importante, la plus créatrice d'emplois comparativement aux investissements qu'exigent les activités de récréation selon Maurice Renoux ${ }^{(3)}$.

Les flux touristiques annuels prévus sont ensuite traités par un modèle interindustriel de la province (ou de la région où est réalisé le grand projet, s'il en existe). Les flux touristiques, par catégories d'hébergements, constituent une demande finale exogène pour le système de comptabilité économique de la province.

Le modèle intersectoriel du Québec ${ }^{(4)}$ par exemple, détermine les conséquences économiques d'un grand projet sur plusieurs plans:

- les effets directs et indirects de la création d'emploi ainsi que les salaires et gages avant impôts:

- la valeur ajoutée;

- et certaines recettes fiscales et parafiscales engendrées par l'activité additionnelle créé par un grand projet.

Les résultats du modèle économétrique du
Québec seront d'autant plus satisfaisants que l'identification des dépenses touristiques, qui constituent la demande fiscale exogene à traiter, est détaillée et précise. D'oú la nécessité d'une enquête très détaillée, menée de façon rigoureuse auprès des touristes ou visiteurs de la région ou de la province où se déroule le grand evénement.

Les données exogènes doivent êttre considérées aux prix courants. Les coefficients du modẻle intersectoriel représentent les proportions selon lesquelles se répartissent les flux de pouvoirs d'achat et reflètent le comportement des agents economiques tel qu'il a été observé. Notons que les prix relatifs varient peu d'une manière générale car l'ensemble des prix varient habituellement de la même façon sur un horizon de cinq à dix ans.

Elkin R.D. et Roberts R.S. ${ }^{(5)}$ expliquent pourquoi les méthodes de production ainsi que les prix relatifs sont parfois appelés à changer. Il ne s'agit que de situations d'exception ou d'un nombre restreint de secteurs d'activité à forte progression technologique; ce qui ne semble pas se retrouver souvent dans les secteurs de services tels que les activites touristiques.

Les tableaux intersectoriels sont remaniés et mis à jour chaque dix ou quinze ans. Celui du Québec le fut en $1978^{6}$. Notons que les coefficients de main-d'oeuvre, de taxes indirectes, de fiscalité et de para-fiscalité furent mis à jour pour l'année $1984^{(6)}$.

Les conséquences des dépenses touristiques annuelles estimées sur la création d'emplois additionnels peuvent être complétées par l'obtention de résultats concernant:

- les niveaux d'activité des divers secteurs productifs;

- les demandes totales des différentes catégories de biens et services;

- les différentes catégories de paiement aux facteurs primaires;

- et les revenus des gouvernements.

Les investissements nouveaux susceptibles de satisfaire l'accroissement de l'activité et des dépenses touristiques, peuvent être calculés de façon séparée, par type d'hébergement, sur la base du nombre annuel additionnel de touristes qu'entraînerait le méga-événement. Un calcul précis tiendrait compte de l'offre d'équipements touristiques et de chambres d'hôtels, de motels disponibles ${ }^{(7)}$.

\section{d.2- Les madèles à caractère purement regional}

Les données régionales sont soit regroupées, à partir de statistiques déjà disponibles. auprès d'organismes officiels nationaux soit élaborées de façon empirique, auprès d'organismes privés telles les institutions de commerce de détail. Ces données sont traitées 
pour alimenter un modèle de détermination des conséquences d'une demande autonome sur une économie locale ou régionale. Les effets directs ou indirects des variations des secteurs de la demande finale sont calculés, notamment au plan de l'emploi. L'économie régionale est subdivisée en deux parties:

- les secteurs d'activité qui desservent des marchés autres que le marché régional et dont la production se traduit, au moins en partie, par des exportations;

- et les secteurs d'activité dont la production est absorbée par le marché régional.

Notons que les flux de production sont les seuls considérés dans ce genre de modèle de flux intersectoriel régional. Les flux importés en sont écartés afin de se soustraire a la contrainte d'utilisaton des coefficients nationaux.

Les firmes exportatrices sont les locomorives qui favorisent le mouvement des secteurs industriels dont les ventes s'effectuent exclusivement sur le plan régional. L'accroissement des activités de celles-là entraîne une augmentation de la production, des ventes et de l'emploi de celles-ci. Plus l'activité d'un hótel destiné à satisfaire les besoins de logement et en restauration de touristes nonrésidents est élevé, plus l'emploi des résidents sera élevế ainsi que leurs dépenses et leurs achats de biens et services dans l'économie régionale.

Le nombre total d'emplois de chaque secteur industriel est subdivisé entre la main-d'oeuvre destinée à produire pour le marché local et celle affectée à la satisfaction des besoins d'exportation. L'estimation du multiplicateur d'emploi s'obtient par le rappori de l'emploi total, par période, d'une région à l'emploi des seules activités exportatrices pour la même période. Un multiplicateur des revenus est estimé de la même façon en divisant les revenus totaux de la région par les revenus engendrés par la production exportée dont font partie les dépenses touristiques comme demande exogène.

L'avantage de la démarche d'auteurs comme

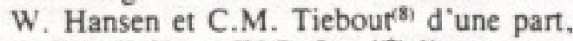
de R.J. Kalter et W.B. Lord'19 d'autre part. réside dans une collecte de données souvent déjà disponibles, plus simples à traiter et moins coûteuses que les méthodes plus laborieuses d'estimation de coefficients locaux. Il faut cependant noter quelques faiblesses à ces modèles dont celle de l'hypothèse d'un méme multiplicateur global pour l'ensemble de la région. Or, les multiplicateurs peuvent ètre différents d'une industrie à l'autre. Par ailleurs, la croissance et le développement d'une région peuvent trouver leurs origines ailleurs que dans les exportations.

Notons qu'un modèle de relations interindustrielles constitue le cadre d'analyse de la base économique des régions métropolitaines et de leurs sources de revenus. Roland Arte ${ }^{i 6 h}$ a construit un modèle d'échanges interindustriels pour présenter et expliquer de façon assez détaillée la base économique des métropoles américaines et leur contribution au produit national brut. Ce genre de modèle a servi d'inspiration pour les études menées par Brian Archer ${ }^{\prime \prime \prime}$ sur les effets des dépenses louristiques en matière de création de revenus, dans une région, en fonction de profils des dépenses de consommation des résidents. Un ensemble de multiplicateurs des dépenses touristiques fut calculé sur le plan régional dont des multiplicateurs de revenus et des multiplicateurs d'emploi. Les études effectuées par Archer en 1977 et en 1982 constituent une excellente analyse approfondie et détaillée des qualités et des limites d"un ensemble de multiplicateurs.

Notons qu'une enquête permet de déterminer les dépenses touristiques, ventilées selon les catégories de touristes. Des structures détaillées de dépenses sont considérées, dont essentiellement une présentation par type de dépenses de consommation. Archer s'est assuré d'un degré de désagrégation assez poussé de la demande des ménages d'une part et a traité les éléments de la demande des ménages en les séparant des coefficients reliant les secteurs industriels d'autre part. L'analyse des effets d'une dépense touristique d'importance est d'autant plus précise que les fuites du système sont bien délimitées. Ces fuites représentent la proportion de dépenses des résidents affectée à l'acquisition de produits et de services importés, l'épargne des résidents et les impóts et taxes quils payent.

Notons que le nombre d'emplois crées, par le flux touristique annuel croissant, peut être déterminé sur la base des proportions ou relations techniques existantes entre le capital employé et la main-d oeuvre utilisée. dans les différentes branches d'activité du tourisme.

Le calcul du multiplicateur d'emploi ${ }^{\text {[12) }}$ correspondant à chaque branche d'activité permet d'établir le nombre d'emplois nouveaux crees:

$$
\mathrm{Nq}=\mathrm{Knq} \cdot \mathrm{Qq}
$$

et

$\lim \mathrm{dt} \rightarrow \frac{0 \mathrm{dNq}}{\mathrm{dt}}=\mathrm{Knq} \cdot \frac{\mathrm{dQq}}{\mathrm{dt}}=\mathrm{knq} \cdot \mathrm{Iq}(5)$ d'où

$\mathrm{K} n=\frac{\mathrm{Nq}}{\mathrm{Qq}}$ (multiplicateur d'emploi moyen) et

$$
\begin{aligned}
& \mathrm{dKn}=\lim \mathrm{dQq} \rightarrow \frac{\mathrm{odNq}}{\mathrm{dQq}} \\
& \text { (multiplicateur d'emploi marginal) }
\end{aligned}
$$

$\mathrm{Nq}=$ le nombre total d'emplois de la branche d'activité " $q$ " reliée au tourisme dans la zone périphérique du méga-projet.

Knq $=$ le multiplicateur d'emplois propre a la branche d'activité " $\mathrm{q}$ ".

$Q q=$ le stock de capital de la branche d'activitế " "q".

Iq = I'investissement de la branche d'activité "q".

\section{Les coûts}

Les catégories suivantes de coûts sont en général retenues dans le cadre d'une analyse de l'impact d'un grand événement touristique comme la création et l'aménagement d'un parc national au Canada:

a) Le coût du rachat des droits sur des richesses ou des ressources

Le gouvernement de la province où se situe le grand événement touristique doit remettre au gouvernement fédéral canadien toutes sortes de droits sur des ressources ou des terres nécessaires à la création et à l'aménagement du parc national. Les dépenses d'acquisition de ces droits constituent un coût pour la province et doivent faire partie d'une analyse coûts-bénéfices. Si l'opération de rachat des droits sur des richesses ou des ressources entraine une perte de revenus futurs, par l'arrét d'une exploitation donnée, il faut en tenir compte parmi les coûts que la province doit accepter dans le cadre du projet de réalisation du parc national.

b) Les couts d'option (ou couts d"'opportunite" ")

Les coùts d'option doivent être intégrés à l'analyse coûts-bénéfices lorsque des ressources affectées aux besoins exclusifs du fonctionnement du parc national peuvent être utilisées ailleurs et dans d'autres activités.

c) Le traitement des coûts de dépenses en capital et de depenses d'exploitation

La province doit affecter des ressources ou des investissements pour relier le parc national aux autoroutes nationales. Par contre. la province réalise des économies annuelles d'entretien car le gouvemement fédéral canadien assure désormais, à sa place, cette catégorie de frais ou de coutts liés aux voies de communications internes du parc. L'investissement total affecté à la liaison entre un parc national et les autoroutes nationales est converti en coûts annuels, selon le taux d'actualisation ou taux d'escompte social. afin de comparer les dépenses en capital aux économies annuelles d'entretien.

Le coút annuel net pour la province concernée s'obtient par la différence entre le coúts annuel de l'investissement et les économies annuelles de dépenses d'entretien. 


\section{Le taux social d'actualisation}

Le choix du taux d'actualisation de la valeur des services et des flux monétaires engendrés par un événement touristique revêt une importance particulière. On ne fera pas état de la controverse théorique sur la détermination du taux social d'actualisation. Le lecteur pourra se référer à $\mathrm{F}$. Rassi à cet égard. Nous retiendrons les critères et modalités $\mathrm{d}^{\text {tapplication }}{ }^{(13)}$ les plus couramment adoptes.

Une estimation inexacte du taux d'escompte des bénéfices d'un événement touristique peut avoir des conséquences défavorables pour les activités concernées et peut aussi entraîner des distortions préjudiciables à l'affectation des ressources productives. La plupart des dépenses importantes d'un événement de nature publique sont réalisées au début de la vie du projet tandis que les bénéfices s'étendent sur plusieurs années et parfois sur un avenir lointain (quinze à vingtcinq ans). La proportion relative du rapport bénéfice/coût a tendance à baisser avec un taux d'actualisation élevé.

Les bénéfices de la récréation et de la préservation procurés par la création de parcs nationaux sont associés à des services nonmarchands. Ils pourraient ềtre actualisés selon une moyenne des taux de rendement à long terme sur les obligations du gouvernement fédéral canadien.

Les bénéfices et les coûts découlant de projets d'investissement tels les hồtels, restaurants et entreprises de transport devraient être actualisés selon le taux d'escompte utilisé par le secteur privế pour la me̊me classe de risque (F. Rassi, page 19 , référence $\left.{ }^{(13)}\right)$.

Les bénéfices et les coûts découlant de projets d'investissements publics, pour lesquels il n'existe pas d'activité équivalente dans le secteur privé, devraient être actualisés selon une moyenne du taux de rendement à long terme sur les obligations du gouvernement fédéral canadien majoré d'une prime de risque. Notons que le coût du capital ou taux d'actualisation est toujours calculé après impôt.

\section{Conclusion}

La plupart des événements touristiques font l'objet d'analyses quantitatives plutót que qualitatives. L'analayse coûts-bénéfices ainsi que les modèles interindustriels sont largement utilisés pour évaluer les conséquences multiples de l'implantation d'un projet touristique notamment sur les plans de l'investissement et de l'emploi. Selon l'auteur de ce texie ${ }^{1 / 4}$, " "il s"agit d"une étude d'impact économique dont l'objet est de déterminer les effets d'entrainement directs et indirects à partir d'une ventilation détaillée des dépenses, à la suite d'une enquête sur place" . L'auteur (4) ajoute que "les effets économiques peuvent alors être précisés et délimités non seulement sur le plan macro-économique mais aussi du point de vue micro-économique de la firme. Aux analyses d'impact global peuvent être ajoutées les études de faisabilité et de rentabilité des dépenses d'investissement d'une entreprise en tant que telle ainsi que les anlayses des différentes sources de financement et de leurs proportions susceptibles de minimiser le coût du capital ou coût de financement ${ }^{*}$.

Le processus de décision est grandement amélioré, au niveau gouvernemental comme au niveau de l'entreprise, lorsque les études d'impact économique sont rigoureuses et pertinentes. Il faut surmonter de nombreuses difficultés de nature statistique, analytique et normative pour atteindre une qualité d'analyse appréciable. Aline Royer ${ }^{(15)}$ procède à une excellente revue critique d'études d'évaluation économique d'événements à caractère touristique au Québec. L'auteur analyse l'évaluation de plusieurs événements touristiques québécois importants sur le plan méthodologique, quant à leurs forces et faiblesses et quant à leurs impacts. La surestimation des résultats constitue un des principaux écueils à éviter dans les études d'impact des grands événements touristiques selon les résultats de recherches et d'analyses établis par Aline Royer. $f$

\section{Notes explicatives}

(1) RITCHIE, J.R. Brent, Assessing the impast of Hallmark Evenis: Conceptual and Fesearch /s. sues, Journal of Travel Research, Vol. 23, No 1. été 1994 .

(2) ROVELSTAD, James M., Model Building and Simulation, in Travel. Tourism and Hospitality Research, edité par J.A. Brent, Richie et C.R. Goeldner, John Wiley and Sons. Toronto. 1978, pp. $449-458$

(3) RENOUX, Maurice, Techniques Econometriques de prevision de la demande touristique et de leur integration dans un systeme décisionnel. Minis. tere du Tourisme, de la Chasse et de la Péche, Service de la Recherche, Québec, Canada, 1972.

(4) RASSI, Faouzi, Les retemberes socioéconomiques d'un développement touristique, Service de la recherche, Ministére du Tourisme, de lat Chasse et de la Páche, Gouvernement du Que. bec, P.Q., Canada, 1975; voir aussi:

- Rapport Intérimaire sur le Systeme de comptabilité Economique du Québec, Le Système et son fonetionnement. volume 1, juillet 1967. Le Système de Comptabilité Economique du Québec: Les Utilisations, volume III: Bureau de la Statistique du Quebec (BSQ); Laboratoire d'Econométrie, Université Laval, Quebec, P.Q., Canada, février 1970.

- Le Systeme de Comptabilite Economique du Québec: Le traitement des depenses de consommation, par Ronald Carré, Division de la recherche. BSO, juin 1971,

- Le Systéme de Comptabilité Economique du Québee: Quelques résultats, par Reeal Marshall, Division de la recherche, BSO, dans la revue Statistiques, Vol. Vill, No 1, 1969.

- Le Tableau Économique du Quebec, 1966, par Jacques Lefort et Real Marchall, Division de la recherche. BSO, dans ta revue Statistiques, Vol. XI, No 1, 1972.

(5) ELKIN, R.D. en ROBERTS, R.S., Evaluating the Human Resource (Employment) Requirements and impacts of Tourism Developments, dans Travel, Tourism and Hospltality Research, a Handbook for Managers and Researchers, édite par J.R. Brent Ritchie et C.R. Goeldner, pp. 363.372, John Wiley and Sons, New York, 1967.
(6) GILBERT Martin, Le Tableau d'entrées-sorties du Quebec pour 1978, Le Modale Intersectoriel ef ses Applications, Bureau de la Statistique du Québec (BSQ), Gouvernement du Québec, P.Q. Canada, 1986.

(7) Voir pour plus de détals F. Rassi, op. eit..

(8) HANSER, W. Lee et Charles M. TIEBOUT, An $/ n$ tersectoral Flows Ans/ysis of the California Economy, The Review of Economics and Statis. tics, Vol. XLV. November 1963. No 4; voir aussi Markets for Californis Products des memes auteurs cintes ci-dessus; voir aussi de C.H. TIEBOUT. The Community Economic Base Study, Committee for Economic Development, Washington, D.C. 1962.

(9) KALTER, Robert J. et W.B. LORD, Measurement of the impact of Recreation Investments on a Local Economy, American Joumal of Agricultural Economics, Vol. 50, No 2, May 1968.

(10) ARTLE, Roland, On Some Methods and Problems in the Study of Metropolitan Econom. ics, dans Regional Seience Association, Paper VIII. Hague Congress, 1961.

(11) ARCHER, Brian, The Impsct of Domestic Teurism, Bangor Oecasional Papers in Econom. ics, No 2, University of Wales Press, 1973. pp. 51-57; voir aussi du meme auteur: Tourism Multipliers: The State of the Art, Bangor Occasional Papers in Economics, No 11. University of Wales Press, 1977. Voir aussi du meme auteur, Value of Multiplicators and their Policy Implications, communication donnée a l'Univergite de Surtey, Guilford, Grande-Bretagne en 1982 dans le cadre du Surrey International Conference on Trends in Tourism Planning and Development.

(12) HIGGINS, B., MARTIN, F et A. REYNAUD, Les orientations du developpement diconomique régional dans la province de Qubbec, Ministere de l'Expansion économique régionale, Otrawa, Canada, 1972.

(13) RASSI, Faouzi, Le taux social d'actualisation et le choix des investissements publics, Document de travail No $77-16$. Faculte des Sciences de l'Administration, Université Laval, Québec. Canada, 1977, 51 pages.

(14) RASSI, Faouzi, Modebles de prévision et impact maero- beonomique d'une demande finale exogène ou d'un mega-evenement, Revue de Tou. risme, No 4, octobre-décembre 1987. Berne.

(15) ROYER, Aline, L'bvaluation deonemique d'tevt. nements à caractère touristique, Depertement des Sciences Economiques, Faculte des Arts et des Sciences, Université de Montréal, memoire de thème Maitre es Sciences, 463 pages, 1983. 